\title{
Transversal Chaos Spaces and Asymptotic Fixed Points
}

\author{
Milan R. TASKOVIĆ
}

\begin{abstract}
This paper continues the study of the transversal spaces. In this sense we formulate a new structure of spaces which we call it transversal chaos spaces. Also, this paper presents an extended asymptotic fixed point theory.
\end{abstract}

\section{INTRODUCTION AND HISTORY}

The notion of distance $d(x, y)$ between points $x$ and $y$ is very old and is essention connection with measurement. Certainly, the notion is present in works of $\mathrm{T}$ h a l e s of Milet (circa 624 B.C.-546 B.C.), one of the seven wise men of the Antic Greece, the first man who predicted the eclipse of the sun (for the year 585 B.C.); he performed various calculations with distance and angles.

A perpetual monument to the old notion of distance is the Pythagoras Theorem on triangles of $\mathrm{P} \mathrm{y} \mathrm{thagoras}$ of $\mathrm{Sam}$ os (circa 560 B.C. - 480 B.C.). In fact, this theorem was already known in Babylon at the time of $\mathrm{K}$ in g $\mathrm{H} \mathrm{a} \mathrm{m} \mathrm{m} \mathrm{u} \mathrm{r} \mathrm{a} \mathrm{b} \mathrm{i}$ (circa 1728 B.C. - 1686 B.C.).

Presumably, however, it was a mathematician of the Pythagorean school who first proved the Pythagorean theorem. This theorem appears as Proposition 47 in Book I of Euclid's Elements (300 B.C.).

The concept of an abstract metric space, introduced by M. Fr é c h e t in 1905, furnishes the common idealization of a large number of mathematical, physical and other scientific constructs in which the notion of a distance appears.

The objects under consideration may be most varied. The may be points, functions, sets, and even the subjective experiences of sensations. A generalization which was first introduced by K. M e n g e r in 1942 and, following him, is called a statistical metric space.

In 1934 Đ. K u r e p a defined pseudodistancional spaces, with the nonnumerical distance, which play an important role in nonlinear numerical analysis (see: L.

2000 Mathematics Subject Classification. Primary: 54E15, 47H10, 05A15. Secondary: $54 \mathrm{E} 35,54 \mathrm{H} 25$.

Key words and phrases. General ecart, distance, Fréchet's spaces, Kurepa's spaces, Menger's spaces, transversal spaces, transversal probabilistic spaces, transversal intervally spaces, transverse, bisection functions, fixed points, intervally contractions, probabilistic contractions, transversal chaos spaces, asymptotic fixed point theory, transversal spaces with nonnumerical transverse, asymptotic behavior in springs of spaces. 
Collatz [7]). After that several authors investigated the distance functions taking values in partially ordered sets (A. A p p e r t, M. Fr é c h e t, J. C o l m e z, R. D o s s, Ky Fan, and others in the year's 40's and 50's).

Concept of transversal spaces where introduced in 1998 by Tasković as a nature extension of Fréchet's, Kurepa's, and Menger's spaces in the following sense. The transversal spaces play an important role in nonlinear functional analysis as and in numerical analysis.

Let $X$ be a nonempty set and let $P:=(P, \preccurlyeq)$ be a partially ordered set. The function $\rho: X \times X \rightarrow P$ is called an upper transverse on $X$ (or upper transversal) iff: $\rho[x, y]=\rho[y, x]$, and if there is an upper bisection function $g: P \times P \rightarrow P$ such that

$$
\rho[x, y] \preccurlyeq \sup \{\rho[x, z], \rho[z, y], g(\rho[x, z], \rho[z, y])\}
$$

for all $x, y, z \in X$. A transversal upper space is a set $X$ together with a given upper transverse on $X$. We call that an element $\zeta \in P$ is a spring of transversal upper space $X$ iff: $\rho[x, y]=\zeta$ if and only if $x=y$.

Let $k=\aleph_{\alpha}(\alpha \geqslant 0)$ be a regular cardinal. Call a topological space $X$ an upper $k$ transversal space or a $g\left(D_{\alpha}\right)$-space if there exists $\rho: X \times X \rightarrow \omega_{\alpha} \cup\left\{\omega_{\alpha}\right\}:=W$ such that $\rho[x, y]=\omega_{\alpha}$ if and only if $x=y, \rho[x, y]=\rho[y, x]$, and if there is $g: W \times W \rightarrow W$ such that (A) for all $x, y, z \in X$. We notice, Fréchet's spaces are important examples of upper $k$-transversal spaces.

Open problem 1. Does for every regular cardinal $k \geqslant \aleph_{\alpha}$ there exists an upper $k$-transversal nonlinearly orderable topological space? Does some of upper transversal spaces have the fixed point property?

Let $X$ be a nonempty set and we chosen an upper bisection function $g:\left(\mathbb{R}_{+}^{0}\right)^{2} \rightarrow$ $\mathbb{R}_{+}^{0}:=[0,+\infty)$ defined in the following sense by

$$
g(s, t)=\psi(s)+\tau t \quad\left(\tau \geqslant 1, \psi: \mathbb{R}_{+}^{0} \rightarrow \mathbb{R}_{+}^{0}\right)
$$

for a self-map $\psi$ with the property $\psi(x) \rightarrow 0(x \rightarrow 0)$, then $X$ is an example of transversal upper space, which where introduced in 1974 by M. C i c c h e s e. Special case of this spaces recently in 1998 is considered S. C z e r w i k.

In connection with the preceding, let $P:=(P, \preccurlyeq)$ be a partially ordered set. The function $\rho: X \times X \rightarrow P$ is colled a lower transverse on $X$ (or lower transversal) iff: $\rho[x, y]=\rho[y, x]$ and if there is a lower bisection function $d: P \times P \rightarrow P$ such that

$$
\inf \{\rho[x, z], \rho[z, y], d(\rho[x, z], \rho[z, y])\} \preccurlyeq \rho[x, y]
$$

for all $x, y, z \in X$. A lower transversal space is a set $X$ together with a given lower transverse on $X$. We call that an element $J \in P$ is a spring of transversal lower space $X$ iff: $\rho[x, y]=J$ if and only if $x=y$.

Let $k=\aleph_{\alpha}(\alpha \geqslant 0)$ be a regular cardinal. Call a topological space $X$ a lower $k$-transversal space or $d\left(D_{\alpha}\right)$-space if there exists the function $\rho: X \times X \rightarrow$ $\omega_{\alpha} \cup\left\{\omega_{\alpha}\right\}:=W$ such that: $\rho[x, y]=\omega_{\alpha}$ if and only if $x=y, \rho[x, y]=\rho[y, x]$, and if there is $d: W \times W \rightarrow W$ such that (B) for all $x, y, z \in X$.

Open problem 2. Does for every regular cardinal $k \geqslant \aleph_{\alpha}$ there exists a lower $k$ transversal nonlinearly orderable topological space? Does some of lower transversal spaces have the fixed point property? 
We notice, in connection with this problem, that work of Đ. K u r e p a in 1963 is very important, where there is result that for every regular cardinal $k \geqslant \aleph_{0}$ there exists a $k$-metrizable (i.e., an $D_{\alpha}$-space) nonlinearly orderable topological space. A proof of this result was exhibit by S. To d o r č e vi ć in 1981.

Karl Menger initiated the study of probabilistic metric spaces in 1942. A probabilistic metric space in which the "distance" between any two points is a probability distribution function. Every Menger's space is a lower transversal space (see: Taskovi c 40]). The possibility of defining such notions as limit and continuity in an arbitrary set is an idea which undoubtedly was first put forward by $\mathrm{M} \mathrm{a} \mathrm{u} \mathrm{-}$ r i c e Fr é c h e t in 1904, and developed by him in his famous doctoral dissertation 1905.

In 1934 Đuro Kurepa introduced the notion of a pseudo-metric space: and in 1936 also Đ. K u r e p a introduced, for a given ordinal $\alpha$, the notion of $\left(\triangle^{\alpha}\right)$ or $\left(D_{\alpha}\right)$ as the class of pseudo-metric spaces. The case $\alpha=0$ coincides with the class of metric spaces.

A special feature in the former notions (of Fréchet and Kurepa) is the "triangular relation" occurring in the elementary geometry and in many other cases.

At the same time, Fréchet consider instead of triangular relation, apparently weaker, regularity condition: There exists a self-map $f$ of $\mathbb{R}_{+}:=(0,+\infty)$ into itself such that $f(x) \rightarrow 0(x \rightarrow 0)$ and that for any triple $(a, b, c)$ of elements of $X$ one has $\rho(a, b)<x$ and $\rho(b, c)<x$ implies $\rho(a, c)<f(x)$.

Fréchet remarked that metric spaces $(X, \rho)$ and preceding spaces $(X, \rho, f)$ with the regularity condition have similar properties. In 1910 he asked whether this two classes of spaces should be the same. C h it t e $\mathrm{n}$ d e $\mathrm{n}$ in 1917 confirmed this conjecture. A simple proof was exhibited by $\mathrm{Fr}$ in k in 1937.

We remarked that an important example of upper transversal spaces is also and every Fréchet's space with the regularity condition. For this an upper bisection function $g:\left(\mathbb{R}_{+}^{0}\right) \rightarrow\left(\mathbb{R}_{+}^{0}\right)$ can be defined by $g(s, t)=\max \{x, f(x)\}$.

On the other hand, let $\tau=\omega_{\mu}$ be a regular cardinal number, $X$ a set, and $(G,+, \preccurlyeq)$ a linearly ordered abelian group with cofinality $\operatorname{cof}(G)=\omega_{\mu}$ at the identity element $0 \in G$ (which means that 0 is the infimum of strictly decreasing $\tau$-sequence $\left.\left\{x_{\alpha}: \alpha \in \tau\right\} \subset G \backslash\{0\}\right)$. An $\tau$-metric on $X$ is a function $\rho: X \times X \rightarrow G$ which satisfies all the metric axioms (i.e., $\rho[x, y]=0$ if and only if $x=y, \rho[x, y]=\rho[y, x]$ and $\rho[x, y] \preccurlyeq \rho[x, z]+\rho[z, y])$.

This definition of spaces $X$ was given by $\mathrm{R}$. S i korski in 1950 using the name $\omega_{\mu}$-metrizable topological space (if its topology can be induced by some $\omega_{\mu}$-metric on $\left.X\right)$.

Call, for $k=\aleph_{\alpha}(\alpha \geqslant 0)$, a topological space $X$ a $k$-metrizable space or a $D_{\alpha}$-space if there exist $\rho: X \times X \rightarrow \omega_{\alpha} \cup\left\{\omega_{\alpha}\right\}$ and $\phi: \omega_{\alpha} \rightarrow \omega_{\alpha}$ such that: $\rho(x, y)=\omega_{\alpha}$ if and only if $x=y, \rho(x, y)=\rho(y, x)$, and if $\rho(x, y)>\phi(\xi)$ implies $\rho(x, z)>\xi$. This definition of space $X$ was given by $Đ$. K u r e p a in 1934 .

Obviously, $\omega_{\mu}$-metrizable topological spaces are fundamental examples of upper transversal spaces with the upper bisection function $g: G \times G \rightarrow G$ defined by $g(s, t) ;=s+t$.

Also, $D_{\alpha}$-spaces of . K u r e p a for $\alpha \geqslant 0$ are fundamental examples of lower transversal spaces with the lower bisection function $d: P \times P \rightarrow P$ defined by 
$d(s, t):=\inf \{\xi, \phi(\xi)\}$ for some function $\phi: \omega_{\alpha} \rightarrow \omega_{\alpha}$ and $\xi<\omega_{\alpha}$. A fundamental first example of upper transversal space for the upper bisection function $g:\left(\mathbb{R}_{+}^{0}\right)^{2} \rightarrow \mathbb{R}_{+}^{0}$ defined by $g(s, t):=s+t$ is a metric space.

For further facts on transversal spaces see: Task ovi ć [37]. In the next two sections we give an asymptotic fixed point theory on new transversal chaos spaces.

For other examples of transversal spaces as: transversal intervally spaces, transversal normed spaces, transversal intervally ordered spaces, transversal spaces with nonnumerical transverse, transversal edges spaces, and transversal spring spaces, see: Tasković [40].

\section{Transversal lower chaos spaces}

Let $X$ be a nonempty set. The function $A: X \times X \rightarrow[a, b]$ (or $A$ : $X \times X \rightarrow(a, b])$ for some $-\infty \leq a<b \leq+\infty$ is called a lower chaos transverse on $X$ (or lower chaos transversal) iff: $A(x, y)=b$ if and only if $x=y$ for all $x, y \in X$.

A lower transversal chaos space (or lower chaos space) is a set $X$ together with a given lower chaos transverse $A$ on $X$ denoted its by $X:=$ $(X, A)$. We call that $b \leq+\infty$ is a spring of the space $X:=(X, A)$.

Otherwise, the function $A$ is called a semilower chaos transverse on $X$ (or semilower chaos transversal) iff: $A(x, y)=b$ implies $x=y$ for all $x, y \in X$. A semilower chaos transversal space $X:=(X, A)$ is a set $X$ together with a given semilower chaos transverse on $X$. For any nonempty set $S$ in the lower chaos transversal space $X$ the trh.diameter of $S$ is defined as

$$
\text { trh. } \operatorname{diam}(S):=\inf \{A(x, y): x, y \in S\} ;
$$

it is an element in $[a, b], A \subset B$ implies trh. $\operatorname{diam}(B) \leqslant \operatorname{trh} \cdot \operatorname{diam}(A)$. The relation $\operatorname{trh} \operatorname{diam}(S)=b$ holds if and only if $S$ is a one point set.

Elements of a lower chaos transversal space will usually be called points. Given a lower chaos transversal space $X:=(X, A)$ and a point $z \in X$, the open ball of center $z$ and radius $r>0$ is the set

$$
A(B(z, r)):=\{x \in X: A(z, x)>b-r\} \text { for } b<+\infty,
$$

and $A(B(z, r)):=\{x \in X: A(z, x)>r\}$ for $b=+\infty$. The convergence $x_{n} \rightarrow x$ as $n \rightarrow \infty$ in the lower chaos transversal space $X:=(X, A)$ means that

$$
A\left(x_{n}, x\right) \rightarrow b \quad(b<+\infty) \text { as } n \rightarrow \infty,
$$

or equivalently, for every $\varepsilon>0$ there exists an integer $n_{0}$ such that the relation $n \geqslant n_{0}$ implies $A\left(x_{n}, x\right)>b-\varepsilon$.

If $b=+\infty$, then the lower chaos convergence $x_{n} \rightarrow x$ as $n \rightarrow \infty$ in the lower chaos transversal space $X:=(X, A)$ means that $A\left(x_{n}, x\right) \rightarrow+\infty$ as $n \rightarrow \infty$ or equivalently, for every $\varepsilon>0$ there exists an integer $n_{0}$ such that the relation $n \geq n_{0}$ implies $A\left(x_{n}, x\right)>\varepsilon$. 
The sequence $\left\{x_{n}\right\}_{n \in \mathbb{N}}$ in the lower chaos transversal space $X:=(X, A)$ is called lower transversal chaotic sequence (or lower chaotic Cauchy sequence) iff for every $\varepsilon>0$ there is an $n_{0}=n_{0}(\varepsilon)$ such that

$$
A\left(x_{n}, x_{m}\right)>b-\varepsilon \text { for all } n, m \geqslant n_{0}
$$

for $b<+\infty$, and $A\left(x_{n}, x_{m}\right)>\varepsilon$ for all $n, m \geq n_{0}$ if $b=+\infty$. Let $X:=$ $(X, A)$ be a lower chaotic transversal space and $T: X \rightarrow X$. We notice, from Taskovi ć [40], that a sequence of iterates $\left\{T^{n}(x)\right\}_{n \in \mathbb{N}}$ in $X$ is said to be lower transversal chaotic sequence if and only if

$$
\lim _{n \rightarrow \infty}\left(\operatorname{trh} . \operatorname{diam}\left\{T^{k}(x): k \geqslant n\right\}\right)=b .
$$

In this sense, a lower transversal chaos space is called lower chaotic complete iff every lower transversal chaotic sequence converges.

Also, a space $X:=(X, A)$ is said to be lower chaotic orbitally complete (or lower chaotic $T$-orbitally complete) iff every lower chaotic transversal sequence which is contained in $\mathcal{O}(x):=\left\{x, T(x), T^{2}(x), \ldots\right\}$ for some $x \in X$ converges in $X$.

A function $f$ mapping $X$ into the reals is $f$-orbitally upper semicontinuous at $p \in X$ iff $\left\{x_{n}\right\}_{n \in \mathbb{N}}$ is a sequence in $\mathcal{O}(x)$ and $x_{n} \rightarrow p(n \rightarrow \infty)$ implies that $f(p) \geqslant \lim$. $\sup f\left(x_{n}\right)$.

Let $X:=(X, A)$ be a lower chaotic transversal space. A mapping $T: X \rightarrow$ $X$ is said to be lower chaotic contraction if there exists an $0 \leqslant \lambda<1$ such that

$$
A(T(x), T(y)) \geqslant \lambda A(x, y)+b(1-\lambda), \quad b<+\infty,
$$

for all points $x, y \in X$. For further facts on the lower chaotic contractions see: Tasković [40].

Let $\left(X, A_{X}\right)$ and $\left(Y, A_{Y}\right)$ be two lower chaotic transversal spaces for $b<$ $+\infty$ and let $T: X \rightarrow Y$. In order, we notice from Tas k ovi ć [40], that $T$ is lower chaotic continuous at $x_{0} \in X$ iff for every $\varepsilon>0$ there exists a $\delta>0$ such that for every $x \in X$ the following relation holds that

$$
A_{X}\left(x_{0}, x\right)>b-\delta \text { implies } A_{Y}\left(T\left(x_{0}\right), T(x)\right)>b-\varepsilon .
$$

A typical first example of a lower chaotic continuous mapping is the lower chaotic contraction on the lower chaos transversal space $X:=(X, A)$. For the further facts on the lower chaotic continuous mappings see: T a s k o v i ć [40].

If $b=+\infty$ and $a \geq 0$, a mapping $T: X \rightarrow X$ is said to be lower chaotic contraction if there exists an $q>1$ such that

$$
A(T(x), T(y)) \geq q A(x, y) \text { for all } x, y \in X .
$$

Then, if $X:=(X, A)$ is a lower chaotic $T$-orbitally complete lower chaos transversal space, then $T$ has a unique fixed point in the lower chaos space $X$. 
Also, in the case $b=+\infty$, let $X:=\left(X, A_{X}\right)$ and $Y:=\left(Y, A_{Y}\right)$ be two lower chaos transversal spaces and let $T: X \rightarrow Y$. The mapping $T$ is lower chaotic continuous at $x_{0} \in X$ iff for every $\varepsilon>0$ there exists $a \delta>0$ such that for every $x \in X$ the following relation holds as

$$
A_{X}\left(x, x_{0}\right)>\delta \text { implies } A_{Y}\left(T(x), T\left(x_{0}\right)\right)>\varepsilon .
$$

A typical first example of a lower chaotic continuous mapping is the mapping $T$ with property (1). For further facts see: Ta s k o v i ć [40].

Let $X$ be a nonempty set, $T: X \rightarrow X$, and let $A: X \times X \rightarrow[a, b]$ (or $A: X \times X \rightarrow(a, b])$ for some $-\infty \leq a<b \leq+\infty$ be a given function. We shall introduce the concept of lower chaos TCS-convergence in a space $X$, i.e., a lower chaos transversal space $X:=(X, A)$ satisfies the condition of lower chaos TCS-convergence iff $x \in X$ and if $A\left(T^{n} x, T^{n+1} x\right) \rightarrow b$ $(n \rightarrow \infty)$ implies that $\left\{T^{n}(x)\right\}_{n \in \mathbb{N}}$ has a convergent subsequence.

Theorem 1. Let $T$ be a mapping of semilower chaos space $X:=(X, A)$ into itself, where $X$ satisfies the condition of lower chaos TCS-convergence. Suppose that for all $x, y \in X$ there exist a sequence of real functions $\left\{\alpha_{n}(x, y)\right\}_{n \in \mathbb{N}}$ such that $\alpha_{n}(x, y) \rightarrow b(n \rightarrow \infty)$ and positive integer $m(x, y)$ such that

$$
A\left(T^{n}(x), T^{n}(y)\right) \geqslant \alpha_{n}(x, y) \text { for all } n \geqslant m(x, y),
$$

where $A: X \times X \rightarrow[a, b]$ for some $-\infty \leq a<b \leq+\infty$. If $x \mapsto A(x, T(x))$ is a T-orbitally upper semicontinuous function or $T$ is orbitally continuous, then $T$ has a unique fixed point $\xi \in X$ and $T^{n}(x) \rightarrow \xi(n \rightarrow \infty)$ for each $x \in X$.

Proof. For $y=T(x)$ from (D) we have that $A\left(T^{n} x, T^{n+1} x\right) \geqslant \alpha_{n}(x, T x)$ for all $n \geqslant m(x, T x))$, and thus we obtain that $A\left(T^{n} x, T^{n+1} x\right) \rightarrow b(n \rightarrow$ $\infty)$. This implies (from lower chaos TCS-convergence) that the sequence of iterates $\left\{T^{n}(x)\right\}_{n \in \mathbb{N}}$ has a convergent subsequence $\left\{T^{n(i)}(x)\right\}_{i \in \mathbb{N}}$ with the limit point $\xi \in X$. Since $x \mapsto A(x, T(x))$ is $T$-orbitally upper semicontinuous, we get

$$
A(\xi, T(\xi)) \geqslant \limsup _{i \rightarrow \infty} A\left(T^{n(i)} x, T^{n(i)+1} x\right)=\limsup _{n \rightarrow \infty} A\left(T^{n} x, T^{n+1} x\right)=b,
$$

which implies that $A(\xi, T(\xi))=b$, i.e., $\xi=T(\xi)$. On the other hand, if $T$ is orbitally continuous the proof of previous fact is trivial. We complete the proof by showing that $T$ can have at most one fixed point. Indeed, if we suppose that $\xi \neq \eta$ were two fixed points, then from (D) we have

$$
A(\xi, \eta)=A\left(T^{n}(\xi), T^{n}(\eta)\right) \geqslant \alpha_{n}(\xi, \eta) \text { for every } n \geqslant m(\xi, \eta) ;
$$

taking limits as $n \rightarrow \infty$ we obtain a contradiction. Thus we obtain that $\xi=\eta$, i.e., $T$ has a unique fixed point in $X$. The proof is complete.

Note that, from the preceding proof of Theorem 1, we can give the following local form of this statement. 
Theorem 2. (Localization of (D)). Let $T$ be a mapping of semilower chaos space $X:=(X, A)$ into itself, where $X$ satisfies the condition of lower chaos TCS-convergence. Suppose that for each $x \in X$ there exist a sequence of real functions $\left\{\alpha_{n}(x, T x)\right\}_{n \in \mathbb{N}}$ such that $\alpha_{n}(x, T x) \rightarrow b(n \rightarrow \infty)$ and positive integer $m(x)$ such that

$$
A\left(T^{n}(x), T^{n+1}(x)\right) \geqslant \alpha_{n}(x, T x) \text { for all } n \geqslant m(x),
$$

where $A: X \times X \rightarrow[a, b]$ for some $-\infty \leq a<b \leq+\infty$. If $x \mapsto A(x, T(x))$ is a $T$-orbitally upper semicontinuous function or $T$ is orbitally continuous, then $T$ has at least one fixed point in $X$.

The proof of this statement is totally analogous with the preceding proof of Theorem 1.

Applications of Theorem 1. In connection with the preceding facts we have the following two "asymptotic" statements for existence of a unique fixed point as applications of Theorem 1 on lower chaos transversal spaces.

Corollary 1. Let $X:=(X, \rho)$, with the continuous lower chaos transverse $\rho \in \mathbb{R}_{+}^{0}$, be a lower chaos complete lower chaos transversal space, $T: X \rightarrow X$ is a continuous function, and $\varphi_{n}:[a, b] \rightarrow[a, b]$ for $n \in \mathbb{N}$ sequence of continuous functions such that for every $n \in \mathbb{N}$ satisfying

$$
\rho\left[T^{n}(x), T^{n}(y)\right] \geqslant \varphi_{n}(\rho[x, y]) \quad \text { for all } \quad x, y \in X ;
$$

and assume also that there exists a function $\varphi:[a, b] \rightarrow[a, b]$ such that for any $t \in[a, b), \varphi(t)>t, \varphi(t)=b$ iff $t=b$, and $\varphi_{n} \rightarrow \varphi(n \rightarrow \infty)$ uniformly on the range of $\rho$. Then $T$ has a unique fixed point in $X$.

Proof. (Application of Theorem 1). Since $\varphi$ is a continuous function such that $\varphi(t)=b$ if and only if $t=b$, we define a function $A: X \times X \rightarrow[a, b]$ by $A(x, y):=\varphi(\rho[x, y])$, and define a sequence of functions $\left\{\alpha_{n}(x, y)\right\}_{n \in \mathbb{N}}$ by $\alpha_{n}(x, y):=\rho\left[T^{n}(x), T^{n}(y)\right]$. For all $x, y \in X(x \neq y)$ we have

$$
\liminf _{n \rightarrow \infty}\left[T^{n}(x), T^{n}(y)\right] \geqslant \liminf _{n \rightarrow \infty} \varphi_{n}(\rho[x, y])=\varphi(\rho[x, y])>\rho[x, y] .
$$

If there exist $z, r \in X$ and $\varepsilon<b$ such that $\liminf _{n \rightarrow \infty} \rho\left[T^{n}(z), T^{n}(r)\right]=$ $b-\varepsilon$, then there exists $k \in \mathbb{N}$ such that $\varphi\left(\rho\left[T^{k}(z), T^{k}(r)\right]\right)>b-\varepsilon$, because $\varphi$ is continuous and $\varphi(b-\varepsilon)>b-\varepsilon$. This implies that

$$
\begin{aligned}
& \liminf _{n \rightarrow \infty} \rho\left[T^{n}(z), T^{n}(r)\right]=\liminf _{n \rightarrow \infty} \rho\left[T^{n}\left(T^{k} z\right), T^{n}\left(T^{k} r\right)\right] \geqslant \\
\geqslant & \liminf _{n \rightarrow \infty} \varphi_{n}\left(\rho\left[T^{k}(z), T^{k}(r)\right]\right)=\varphi\left(\rho\left[T^{k}(z), T^{k}(r)\right]\right)>b-\varepsilon,
\end{aligned}
$$

which is a contradiction. Thus $\rho\left[T^{n}(x), T^{n}(y)\right] \rightarrow b$ as $n \rightarrow \infty$ for all $x, y \in X$, which implies that all sequences of iterates, defined by $T$, are equi-convergent in lower chaos transversal space $(X, \rho)$. This means that $\alpha_{n}(x, y) \rightarrow b(n \rightarrow \infty)$ as in Theorem 1. Since $\varphi(t)=b$ iff $t=b$, from $A(x, y)=\varphi(\rho[x, y])=b$ it follows that $\rho[x, y]=b$, i.e., $x=y$. Also, $x \mapsto$ $A(x, T(x)):=\varphi(\rho[x, T(x)])$ is $T$-orbitally upper semicontinuous, because $T$, 
$\varphi$, and $\rho$ are continuous. Since $X$ satisfies the condition of lower chaos TCSconvergence ( $X$ is a lower chaos complete lower chaos transversal space), applying Theorem 1, we obtain that $T$ has a unique fixed point in $X$. The proof is complete.

Corollary 2. Let $X:=(X, \rho)$, with the continuous lower chaos transverse $\rho \in \mathbb{R}_{+}^{0}$, be a lower chaos complete lower chaos transversal space, $T: X \rightarrow X$ is a continuous function, and $\varphi_{n}:[a, b] \rightarrow[a, b]$ for $n \in \mathbb{N}$ sequence of continuous functions such that for every $n \in \mathbb{N}$ satisfying

$$
\begin{gathered}
\rho\left[T^{n}(x), T^{n}(y)\right] \geqslant \\
\geqslant \min \left\{\varphi_{n}(\rho[x, y]), \varphi_{n}(\rho[x, T x]), \varphi_{n}(\rho[y, T y]), \varphi_{n}(\rho[x, T y]), \varphi_{n}(\rho[y, T x])\right\}
\end{gathered}
$$

for all $x, y \in X$; and assume also that there exists function $\varphi:[a, b] \rightarrow[a, b]$ such that for any $t \in[a, b), \varphi(t)>t, \varphi(t)=b$ iff $t=b$, and $\varphi_{n} \rightarrow \varphi$ $(n \rightarrow \infty)$ uniformly on the range of $\rho$. Then $T$ has a unique fixed point in $X$.

Proof. (Application of Theorem 1). Since $\varphi$ is a continuous function such that $\varphi(t)=b$ if and only if $t=b$, we define a function $A: X \times X \rightarrow[a, b]$ by $A(x, y):=\min \{\varphi(\rho[x, y]), \varphi(\rho[x, T x]), \varphi(\rho[y, T y]), \varphi(\rho[x, T y]), \varphi(\rho[y, T x])\}$

and define a sequence of functions $\left\{\alpha_{n}(x, y)\right\}_{n \in \mathbb{N}}$ by

$$
\begin{gathered}
\alpha_{n}(x, y):= \\
=\min \left\{\rho\left[T^{n} x, T^{n} y\right], \rho\left[T^{n} x, T^{n+1} x\right], \rho\left[T^{n} y, T^{n+1} y\right], \rho\left[T^{n} x, T^{n+1} y\right], \rho\left[T^{n} y, T^{n+1} x\right]\right\},
\end{gathered}
$$

then $A$ and $\alpha_{n}(x, y)$ satisfy all the required hypotheses (as in the proof of Corollary 1) in Theorem 1 . Thus $T$ has a unique fixed point in $X$. The proof is complete.

Further applications of Theorem 1. In further we give the following examples of Theorem 1 as some examples of lower chaos transversal spaces.

Example 1. (Metric spaces). A fundamental first example of lower chaos transversal space is a metric space. Indeed, if $(X, q)$ is a metric space, then for the lower chaos transverse $\rho: X \times X \rightarrow[a, b] \subset \mathbb{R}_{+}^{0}$ for $a<b$ defined by

$$
\rho[x, y]=\frac{(a-b) q[x, y]}{1+q[x, y]}+b
$$

for all $x, y \in X$ we have that $(X, \rho)$ is an example of a lower chaos transversal space. In general, every metric space is an example of a lower chaos transversal space.

Example 2. (Lower probabilistic spaces). A mapping $F: \mathbb{R} \rightarrow \mathbb{R}_{+}^{0}$ is called a left distribution function if it is nondecreasing, left-continuous with $\inf F=0$ and $\sup F=1$. We will denote by $\mathcal{L}$ the set of all left distribution functions. We shall denote the left distribution function $\mathcal{L}(p, q)$ by $F_{p, q}(x)$, whence $F_{p, q}(x)$ will denote the value of $F_{p, q}$ at $x \in \mathbb{R}$. 
An example of lower chaos transversal space is a lower probabilistic space which is a nonempty set $X$ together with the functions $F_{p, q}(x)$ with the following properties: $F_{p, q}(x)=F_{q, p}(x), F_{p, q}(0)=0$,

$$
F_{p, q}(x)=1 \text { for } x>0 \text { if and only if } p=q \text {, }
$$

and if there is a nondecreasing functions $\tau:[0,1]^{2} \rightarrow[0,1]$ with the property $\tau(t, t) \geqslant t$ for all $t \in[0,1]$ such that

$$
F_{p, q}(x+y) \geqslant \tau\left(F_{p, r}(x), F_{r, q}(y)\right)
$$

for all $p, q, r \in X$ and for all $x, y \geqslant 0$. Then, from (2), we immediately obtain that every lower probabilistic space, for $\rho[p, q]=F_{p, q}(x): X \times X \rightarrow[0,1]$ is a lower chaos transversal space.

Example 3. (Lower parametric chaos transversal spaces). In connection with the preceding facts, the function $N: X \times X \times \mathbb{R} \rightarrow[a, b]$ for some $-\infty \leq a<b \leq$ $+\infty$ is called a lower parametric chaos transverse on $X$ (or a lower parametric chaos transversal) iff: for some $c \in \mathbb{R}_{+}^{0}$ is $N(u, v, t)=b$ for every $t>c$ if and only if $u=v$, and $\lim _{n \rightarrow \infty} N\left(u, v, x_{n}\right)=b$ for arbitrary nondecreasing sequence $\left\{x_{n}\right\}_{n \in \mathbb{N}}$ in $[c,+\infty)$ with $x_{n} \rightarrow+\infty(n \rightarrow \infty)$.

A transversal lower chaos parametric space is a set $X$ together with a given lower parametric chaos transverse $N: X \times X \times \mathbb{R} \rightarrow[a, b]$ for some $-\infty \leq a<b \leq+\infty$ in notation $X:=(X, N)$. De facto, every transversal lower chaos parametric space $X:=(X, N)$, for $A=N$, is a lower chaos transversal space. For this spaces the following "asymptotic" statement holds.

Corollary 3. Let $X:=(X, N)$ be a transversal lower parametric chaos space with the continuous lower parametric chaos transverse $N \in \mathbb{R}_{+}^{0}, T: X \rightarrow X$ is a continuous function, and $X$ with the condition of lower chaos TCSconvergence. Suppose that there exists a function $\varphi:[c,+\infty) \rightarrow[c,+\infty)$ for some $c \in \mathbb{R}$ satisfying $\varphi(t)>t$ for every $t>c$ and

$$
\lim _{n \rightarrow \infty} \varphi^{n}(t)=+\infty \text { for every } t>c
$$

and such that

$$
N\left(T^{n}(x), T^{n}(y), t\right) \geqslant N\left(x, y, \varphi^{n}(t)\right) \text { for every } n \in \mathbb{N},
$$

for every $t>c$, and for all $x, y \in X$. Then $T$ has a unique fixed point in $X$.

Proof. (Application of Theorem 1). We define a function $A: X \times X \rightarrow$ $[a, b]$ for $-\infty \leq a<b \leq+\infty$ by $A(u, v):=N(u, v, t)$ and define a sequence of functions $\left\{\alpha_{n}(u, v)\right\}_{n \in \mathbb{N}}$ by $\alpha_{n}(u, v):=N\left(u, v, \varphi^{n}(t)\right)$.

Thus $\alpha_{n}(u, v) \rightarrow \lim _{n \rightarrow \infty} N\left(u, v, \varphi^{n}(t)\right)=b$ as $n \rightarrow \infty$ as in Theorem 1. Also, $x \mapsto A(x, T x):=N(x, T x, t)$ is $T$-orbitally upper semicontinuous, because $T$ and $N$ are continuous. Since $A(u, v):=N(u, v, t)=b$ implies $u=v$ for every $t>c$, and since $X$ satisfies the condition of lower chaos TCS-convergence, applying Theorem 1 we obtain that $T$ has a unique fixed point in $X$. The proof is complete. 
We notice that the following "asymptotic" statement holds which is as a special case of the preceding Corollary 3.

Corollary 4. Let $X:=(X, N)$ be a transversal lower parametric chaos space with the continuous lower parametric chaos transverse $N \in \mathbb{R}_{+}^{0}, T: X \rightarrow X$ is a continuous function, and $X$ with the condition of lower chaos TCSconvergence. Suppose that there exists an increasing continuous function $\varphi$ : $[c,+\infty) \rightarrow[c,+\infty)$ for some $c \in \mathbb{R}$ satisfying $\varphi(t)<t$ for every $t \in[c,+\infty)$ such that

$$
N\left(T^{n}(x), T^{n}(y), \varphi^{n}(t)\right) \geqslant N(x, y, t)
$$

for every $n \in \mathbb{N}$, for all $x, y \in X$ and for every $t>c$. Then $T$ has a unique fixed point in $X$.

Proof. Since for the function $\varphi:[c,+\infty) \rightarrow[c,+\infty)$ for some $c \in \mathbb{R}$ there is the inverse function $\varphi^{-1}:[c,+\infty) \rightarrow[c,+\infty)$ with the property (As), thus from (B') we obtain a form of the inequality (E) in the form of the following inequality as

$$
N\left(T^{n}(x), T^{n}(y), t\right) \geqslant N\left(x, y, \varphi^{-n}(t)\right) \text { for } n \in \mathbb{N}
$$

and for all $x, y \in X$. Thus, applying Corollary 3 we obtain this statement as a consequence. The proof is complete.

An essential remark. We notice that the lower parametric transversal chaos spaces are, de facto, also the lower chaos transversal spaces.

\section{Transversal UpPer ChaOs SPACES}

Let $X$ be a nonempty set. The function $A: X \times X \rightarrow[a, b]$ (or $A$ : $X \times X \rightarrow[a, b))$ for some $-\infty \leq a<b \leq+\infty$ is called an upper chaos transverse on $X$ (or lower chaos transversal) iff: $A(x, y)=a$ if and only if $x=y$ for all $x, y \in X$.

An upper transversal chaos space (or upper chaos space) is a set $X$ together with a given upper chaos transverse $A$ on $X$ denoted its by $X:=$ $(X, A)$. We call that $a \geq-\infty$ is a spring of the space $X:=(X, A)$.

Otherwise, the function $A$ is called a semiupper chaos transverse on a nonempty set $X$ iff: $A(x, y)=a$ implies $x=y$ for all $x, y \in X$. A semiupper chaos transversal space $X:=(X, A)$ is a nonempty set $X$ together with a given semiupper chaos transverse $A$ on $X$.

Let $X:=(X, A)$ be an upper chaos transversal space, where $T: X \rightarrow X$, and $A: X \times X \rightarrow[a, b]$ for some $-\infty \leq a<b \leq+\infty$ is a given functional. For $S \subset X$ we denoted trh. $\operatorname{diam}(S)$ as a transversal chaos diameter of $S$, in the sense that

$$
\text { trh. } \operatorname{diam}(S):=\sup \{A(x, y): x, y \in S\},
$$

where $S \subset Y$ implies trh. $\operatorname{diam}(S) \leq$ trh. $\operatorname{diam}(Y)$. 
Elements of an upper chaos transversal space will usually be called points. Given an upper chaos transversal space $X:=(X, A)$, and a point $z \in X$, the open ball of center $z$ and radius $r>0$ is the set

$$
A(B(z, r))=\{x \in X: A(z, x)<a+r\}, \text { for } a>-\infty,
$$

and $A(B(z, r)):=\{x \in X: A(z, x)<r\}$ for $a=-\infty$. The upper chaos convergence $x_{n} \rightarrow x$ as $n \rightarrow \infty$ in the upper chaos transversal space $X:=(X, A)$ means that the following fact holds that

$$
A\left(x_{n}, x\right) \rightarrow a \quad(-\infty<a) \text { as } n \rightarrow \infty,
$$

or equivalently, for every $\varepsilon>0$ there exists an integer $n_{0}$ such that the relation $n \geqslant n_{0}$ implies $A\left(x_{n}, x\right)<a+\varepsilon$.

If $a=-\infty$, then the upper chaos convergence $x_{n} \rightarrow x$ as $n \rightarrow \infty$ in the upper chaos transversal space $X:=(X, A)$ means that $A\left(x_{n}, x\right) \rightarrow-\infty$ as $n \rightarrow \infty$ or equivalently, for every $\varepsilon<0$ there exists an integer $n_{0}$ such that the relation $n \geq n_{0}$ implies $A\left(x_{n}, x\right)<\varepsilon$.

The sequence $\left\{x_{n}\right\}_{n \in \mathbb{N}}$ in the upper transversal chaotic space $X:=(X, A)$ is called upper transversal chaotic sequence (or upper chaotic Cauchy sequence) iff: for every $\varepsilon>0$ there is an $n_{0}=n_{0}(\varepsilon)$ such that

$$
A\left(x_{n}, x_{m}\right)<a+\varepsilon \text { for all } n, m \geqslant n_{0}
$$

for $-\infty<a$, and $A\left(x_{n}, x_{m}\right)<-\varepsilon$ for all $n, m \geq n_{0}$ if $a=-\infty$.

Let $X$ be an upper chaos transversal space and $T: X \rightarrow X$. We notice, from Tas kovi ć [40], that a sequence of iterates $\left\{T^{n}(x)\right\}_{n \in \mathbb{N}}$ in $X$ is said to be upper transversal chaotic sequence if and only if

$$
\lim _{n \rightarrow \infty}\left(\operatorname{trh} . \operatorname{diam}\left\{T^{k}(x): k \geqslant n\right\}\right)=a .
$$

In this sense, an upper transversal chaos space is called upper chaotic complete iff every upper transversal chaotic sequence upper chaotic converges. Also, a space $X:=(X, A)$ is said to be upper chaotic orbitally complete (or upper chaotic T-orbitally complete) iff every upper chaotic transversal sequence which contained in $\mathcal{O}(x)$ for some $x \in X$ upper chaotic converges in $X$.

A function $f$ mapping into the reals is $f$-orbitally lower semicontinuous at $p \in X$ iff $\left\{x_{n}\right\}_{n \in \mathbb{N}}$ is a sequence in $\mathcal{O}(x)$ and $x_{p} \rightarrow p(n \rightarrow \infty)$ implies that $f(p) \leq \lim$. inf $f\left(x_{n}\right)$.

Let $X:=(X, A)$ be an upper chaotic transversal space. A mapping $T: X \rightarrow X$ is said to be upper chaotic contraction if there exists an $0 \leq \lambda<1$ such that

$$
A(T(x), T(y)) \leq \lambda A(x, y)+a(1-\lambda), \quad-\infty<a,
$$

for all points $x, y \in X$. For further facts on the upper chaotic contractions see: Tasković [40].

Let $\left(X, A_{X}\right)$ and $\left(Y, A_{Y}\right)$ be two upper chaos transversal spaces for $a>-\infty$ and let $T: X \rightarrow Y$. In order, we notice, from Tasković [40], 
that $T$ is upper chaotic continuous at $x_{0} \in X$ iff for every $\varepsilon>0$ there exists an $\delta>0$ such that for every $x \in X$ the following relation holds that

$$
A_{X}\left(x_{0}, x\right)<a+\delta \text { implies } A_{Y}\left(T\left(x_{0}\right), T(x)\right)<a+\varepsilon .
$$

A typical first example of an upper chaotic continuous mapping is the upper chaotic contraction on the upper chaos transversal space $X:=(X, A)$. For the further facts on the upper chaotic continuous mappings see: T a s k ović [40].

If $a=-\infty$ and $b \leq 0$, a mapping $T: X \rightarrow X$ is said to be upper chaotic contraction if there exists an $q>1$ such that

$$
A(T(x), T(y)) \leq q A(x, y) \text { for all } x, y \in X .
$$

Then, if $X:=(X, A)$ is an upper chaotic $T$-orbitally complete upper chaos transversal space, then $T$ has a unique fixed point in the upper chaos space $X$.

Also, in the case $a=-\infty$, let $X:=\left(X, A_{X}\right)$ and $Y:=\left(Y, A_{Y}\right)$ be two upper chaos transversal spaces and let $T: X \rightarrow Y$. The mapping $T$ is upper chaotic continuous at $x_{0} \in X$ iff for every $\varepsilon<0$ there exists an $\delta<0$ such that for every $x \in X$ the following relation hold as

$$
A_{X}\left(x, x_{0}\right)<\delta \text { implies } A_{Y}\left(T(x), T\left(x_{0}\right)\right)<\varepsilon
$$

A typical first example of an upper chaotic continuous mapping is the mapping $T$ with property (3). For further facts see: Tas k o v i ć [40].

Let $X$ be a nonempty set, $T: X \rightarrow X$, and let $A: X \times X \rightarrow[a, b]$ (or $A$ : $X \times X \rightarrow[a, b))$ for some $-\infty \leq a<b \leq+\infty$ be a given function. In 1986 Tasković investigated the concept of upper chaos TCS-convergence in a space $X$, i.e., an upper chaos transversal space $X:=(X, A)$ satisfies the condition of upper chaos TCS-convergence iff $x \in X$ and if $A\left(T^{n} x, T^{n+1} x\right) \rightarrow a$ $(n \rightarrow \infty)$ implies that $\left\{T^{n}(x)\right\}_{n \in \mathbb{N}}$ has a convergent subsequence.

Theorem 3. Let $T$ be a mapping of semiupper chaos space $X:=(X, A)$ into itself, where $X$ satisfies the condition of upper chaos TCS-convergence. Suppose that for all $x, y \in X$ there exist a sequence of real functions in the form $\left\{\alpha_{n}(x, y)\right\}_{n \in \mathbb{N}}$ such that $\alpha_{n}(x, y) \rightarrow a(n \rightarrow \infty)$ and positive integer $m(x, y)$ such that

$$
A\left(T^{n}(x), T^{n}(y)\right) \leqslant \alpha_{n}(x, y) \text { for all } n \geqslant m(x, y),
$$

where $A: X \times X \rightarrow[a, b]$ for some $-\infty \leq a<b \leq+\infty$. If $x \mapsto A(x, T(x))$ is a T-orbitally lower semicontinuous function or $T$ is orbitally continuous, then $T$ has a unique fixed point $\xi \in X$ and $T^{n}(x) \rightarrow \xi(n \rightarrow \infty)$ for each $x \in X$.

Proof. For $y=T(x)$ from $(\mathrm{G})$ we have that $A\left(T^{n} x, T^{n+1} x\right) \leqslant \alpha_{n}(x, T x)$ for all $n \geqslant m(x, T x))$, and thus we obtain that $A\left(T^{n} x, T^{n+1} x\right) \rightarrow a$ $(n \rightarrow \infty)$. This implies (from upper chaos TCS-convergence) that the sequence of iterates $\left\{T^{n}(x)\right\}_{n \in \mathbb{N}}$ has a convergent subsequence $\left\{T^{n(i)}(x)\right\}_{i \in \mathbb{N}}$ 
with the limit point $\xi \in X$. Since $x \mapsto A(x, T(x))$ is $T$-orbitally lower semicontinuous, we get

$$
A(\xi, T(\xi)) \leqslant \liminf _{i \rightarrow \infty} A\left(T^{n(i)} x, T^{n(i)+1} x\right)=\liminf _{n \rightarrow \infty} A\left(T^{n} x, T^{n+1} x\right)=a,
$$

which implies that $A(\xi, T(\xi))=a$, i.e., $\xi=T(\xi)$. On the other hand, if $T$ is orbitally continuous the proof of previous fact is trivial. We complete the proof by showing that $T$ can have at most one fixed point. Indeed, if we suppose that $\xi \neq \eta$ were two fixed points, then from $(\mathrm{G})$ we have

$$
a<A(\xi, \eta)=A\left(T^{n}(\xi), T^{n}(\eta)\right) \leqslant \alpha_{n}(\xi, \eta) \text { for every } n \geqslant m(\xi, \eta) ;
$$

taking limits as $n \rightarrow \infty$ we obtain a contradiction. Thus we obtain that $\xi=\eta$, i.e., $T$ has a unique fixed point $\xi \in X$. The proof is complete.

Remarks. We notice that Theorem 3 is a generalization of Caccioppoli's theorem as well as many others.

Also, Theorem 3 is a far-reaching generalization of well-known Kantorovitch's theorem in 1939 year in numerical analysis as and a generalization of all theorems of elementary fixed point theory!? For this see: Ta s k o vi ć [40].

We notice that a variant of this statement (of Theorem 3) has been for the first time proved $\mathbf{2 1}$ years ago in: Tasković [Fundamental elements of the fixed point theory, ZUNS-1986, Theorem 4, p. 170]. Many authors this historical fact are to neglect and to ignore.

Note that, from the preceding proof of Theorem 3, we can give the following local form of this statement.

Theorem 4. (Localization of $(\mathrm{G})$ ). Let $T$ be a mapping of semiupper chaos space $X:=(X, A)$ into itself, where $X$ satisfies the condition of upper chaos TCS-convergence. Suppose that for each $x \in X$ there exist a sequence of real functions $\left\{\alpha_{n}(x, T x)\right\}_{n \in \mathbb{N}}$ such that $\alpha_{n}(x, T x) \rightarrow a(n \rightarrow \infty)$ and positive integer $m(x)$ such that

$$
A\left(T^{n}(x), T^{n+1}(x)\right) \leqslant \alpha_{n}(x, T x) \text { for all } n \geqslant m(x),
$$

where $A: X \times X \rightarrow[a, b]$ for some $-\infty \leq a<b \leq+\infty$. If $x \mapsto A(x, T x)$ is a T-orbitally lower semicontinuous function or $T$ is orbitally continuous, then $T$ has at least one fixed point in $X$.

The proof of this statement is totally analogous with the preceding proof of Theorem 3. A brief suitable proof of this statement may be found in Tasković [40].

Applications of Theorem 3. In connection with the preceding facts we have the following "asymptotic" statements for the existence of a unique fixed point as applications of Theorem 3 on upper chaos transversal spaces.

Corollary 5. Let $(X, \rho)$, with the continuous chaotic transverse $\rho \in \mathbb{R}_{+}^{0}$, be an upper chaos complete upper chaos transversal space, $T: X \rightarrow X$ is 
a continuous function, and $\varphi_{n}:[a, b] \rightarrow[a, b]($ for $n \in \mathbb{N})$ a sequence of continuous functions such that for every $n \in \mathbb{N}$ satisfying

$$
\rho\left[T^{n}(x), T^{n}(y)\right] \leq \varphi_{n}(\rho[x, y]) \quad \text { for all } \quad x, y \in X ;
$$

and assume also that there exists a function $\varphi:[a, b] \rightarrow[a, b]$ such that for any $t \in(a, b], \varphi(t)<t, \varphi(t)=a$ iff $t=a$, and $\varphi_{n} \rightarrow \varphi$ uniformly on the range $\rho$. Then $T$ has a unique fixed point $\xi \in X$ and all sequence of Picard iterates defined via $T$ converges to $\xi$.

Annotations. In connection with this, we notice that in $2003 \mathrm{~W}$. Kirk introduced the notion of asymptotic contraction on a metric space, and proved a fixed point theorem for such contractions of the preceding type (K). De facto, in special case of Corollary 5, for the metric spaces, we obtain Kirk's theorem [17, Theorem 2.1, p. 647].

Proof of Corollary 5. (Application of Theorem 3). Suppose that that all the conditions of Corollary 5 are satisfied. We prove that all conditions of Theorem 3 are satisfied. Let us define $A$ by $A(x, y)=\varphi(\rho[x, y])$, and define a sequence of functions $\left\{\alpha_{n}(x, y)\right\}_{n \in \mathbb{N}}$ by $\alpha_{n}(x, y)=\rho\left[T^{n} x, T^{n} y\right]$ for $x, y \in X$. Since $\varphi(t)<t$ for $t>a$ we get that

$$
A\left(T^{n} x, T^{n} y\right)=\varphi\left(\rho\left[T^{n} x, T^{n} y\right]\right)<\rho\left[T^{n} x, T^{n} y\right]=\alpha_{n}(x, y)
$$

that is that the condition $(\mathrm{G})$ is satisfied. Since $\varphi(t)=a$ iff $t=a$, from $A(x, y)=\varphi(\rho[x, y])=a$ it follows that $\rho[x, y]=a$, i.e., $x=y$. On the other hand, for all $x, y \in X(x \neq y)$ we have

$$
\limsup _{n \rightarrow \infty} \rho\left[T^{n}(x), T^{n}(y)\right] \leqslant \limsup _{n \rightarrow \infty} \varphi_{n}(\rho[x, y])=\varphi(\rho[x, y])<\rho[x, y] .
$$

If there exist $z, r \in X$ and $\varepsilon>0$ such that $\lim _{\sup } \sup _{n \rightarrow \infty} \rho\left[T^{n}(z), T^{n}(r)\right]=$ $a+\varepsilon$, then there exists $k \in \mathbb{N}$ such that $\varphi\left(\rho\left[T^{k}(z), T^{k}(r)\right]\right)<a+\varepsilon$, because $\varphi$ is continuous and $\varphi(a+\varepsilon)<a+\varepsilon$. This implies that

$$
\begin{aligned}
& \limsup _{n \rightarrow \infty} \rho\left[T^{n}(z), T^{n}(r)=\limsup _{n \rightarrow \infty} \rho\left[T^{n}\left(T^{k} z\right), T^{n}\left(T^{k} r\right)\right] \leqslant\right. \\
& \leqslant \limsup _{n \rightarrow \infty} \varphi_{n}\left(\rho\left[T^{k}(z), T^{k}(r)\right]\right)=\varphi\left(\rho\left[T^{k}(z), T^{k}(r)\right]\right)<a+\varepsilon,
\end{aligned}
$$

which is a contradiction. Thus $\rho\left[T^{n}(x), T^{n}(y)\right] \rightarrow a$ as $n \rightarrow \infty$ for all $x, y \in X$, which implies that all sequences of iterates, defined by $T$, are equi-convergent in upper general edges transversal space $(X, \rho)$. This means that $\alpha_{n}(x, y) \rightarrow a(n \rightarrow \infty)$ as in Theorem 3 .

Also, $x \mapsto A(x, T(x)):=\varphi(\rho[x, T(x)])$ is lower semicontinuous, because $T, \varphi$ and $\rho$ are continuous. Since $X$ satisfies the condition of upper chaos TCS-convergence ( $X$ is an upper chaos complete upper chaos transversal space), applying Theorem 3 we obtain that $T$ has a unique fixed point $\xi \in X$ and all Picard iterates converge to $\xi$. The proof is complete. 
Corollary 6. Let $(X, \rho)$, with continuous chaos transverse $\rho \in \mathbb{R}_{+}^{0}$, be an upper chaos complete upper chaos transversal space, $T: X \rightarrow X$ is a continuous function, and $\varphi_{n}:[a, b] \rightarrow[a, b]($ for $n \in \mathbb{N})$ a sequence of continuous functions such that for all $n \in \mathbb{N}$ satisfying

$$
\begin{gathered}
\rho\left[T^{n}(x), T^{n}(y)\right] \leqslant \\
\leqslant \max \left\{\varphi_{n}(\rho[x, y]), \varphi_{n}(\rho[x, T x]), \varphi_{n}(\rho[y, T y]), \varphi_{n}(\rho[x, T y]), \varphi_{n}(\rho[y, T x])\right\}
\end{gathered}
$$

for all $x, y \in X$; and assume also that there exists a function $\varphi:[a, b] \rightarrow[a, b]$ such that for any $t \in(a, b], \varphi(t)<t, \varphi(t)=a$ iff $t=a$, and $\varphi_{n} \rightarrow \varphi$ $(n \rightarrow \infty)$ uniformly of the range of $\rho$. Then $T$ has a unique fixed point $\xi \in X$ and all sequences of Picard's iterations defined by $T$ convergates to $\xi$.

Proof. (Application of Theorem 3). We define a function $A: X \times X \rightarrow[a, b]$ for some $-\infty \leq a<b \leq+\infty$ by the following equality in the form as $A(x, y):=\max \{\varphi(\rho[x, y]), \varphi(\rho[x, T x]), \varphi(\rho[y, T y]), \varphi(\rho[x, T y]), \varphi(\rho[y, T x])\}$ and define a sequence of functions $\left\{\alpha_{n}(x, y)\right\}_{n \in \mathbb{N}}$ by the following equality in the adequate form as the following fact

$$
\begin{gathered}
\alpha_{n}(x, y):= \\
=\max \left\{\rho\left[T^{n} x, T^{n} y\right], \rho\left[T^{n} x, T^{n+1} x\right], \rho\left[T^{n} y, T^{n+1} y\right], \rho\left[T^{n} x, T^{n+1} y\right], \rho\left[T^{n} y, T^{n+1} x\right]\right\} .
\end{gathered}
$$

It is easy to show that $A$ and $\left\{\alpha_{n}(x, y)\right\}_{n \in \mathbb{N}}$ satisfy all the required hypothesis (similarly as in the proof of Corollary 5) in Theorem 3. Applying Theorem 3 we get conclusion of Corollary 6 . This completes the proof.

We notice that Corollary 6 is an asymptotic version of Ivanov's theorem (with the metric spaces) on upper chaos transversal spaces, see: I va n o v [15].

Further applications of Theorem 3. In further we give the following consequences of Theorem 3 as some examples of upper chaos transversal spaces.

Example 4. (Metric spaces). A fundamental first example of upper chaos spaces is a metric space. Indeed, if $(X, d)$ is a metric space, then for the upper chaos transverse $\rho: X \times X \rightarrow[a, b] \subset \mathbb{R}_{+}^{0}$ for $a<b$ defined by

$$
\rho[x, y]=\frac{(b-a) d(x, y)}{1+d(x, y)}+a
$$

for all $x, y \in X$ we have that $(X, \rho)$ is an example of an upper chaos transversal space. In general, every metric space is an example of an upper chaos transversal space.

Example 5. (The extended real line $\overline{\mathbb{R}}$ ). The function $f$ is defined in $\mathbb{R}$ by $f(x)=x /(1+|x|)$ is a bijection on $\mathbb{R}$ on the open interval $(-1,1) \subset \mathbb{R}$, and the inverse mapping $g$ being defined by $g(x)=x /(1-|x|)$ for $|x|<1$. Let $\overline{\mathbb{R}}$ be the set which is the union of $\mathbb{R}$ and two new elements written $+\infty$ and $-\infty$ (points at infinity); then we extend $f$ to a bijection of $\overline{\mathbb{R}}$ onto $[-1,1]$ by putting $f(+\infty)=1$, $f(-\infty)=-1$, and write again $g$ for the inverse mapping. 
We can apply this process described to define $\overline{\mathbb{R}}$ as an upper chaos transversal space by putting for the upper chaos transverse $\rho: \overline{\mathbb{R}} \times \overline{\mathbb{R}} \rightarrow[0,2]$ that is

$$
\rho[x, y]=\left|\frac{x}{1+|x|}-\frac{y}{1+|y|}\right|
$$

for all $x, y \in \overline{\mathbb{R}}$. (We notice that for $x \geqslant 0$ is $\rho[+\infty, x]=1 /(1+|x|)$, and for $x \leq 0$ that is $\rho[-\infty, x]=1 /(1+|x|))$.

Example 6. (Upper probabilistic spaces). A mapping $M: \mathbb{R} \rightarrow \mathbb{R}$ is called upper distribution function if it is nonincreasing, left-continuous with inf $M=$ 0 and $\sup M=1$. We shall denote by $\mathcal{U}$ the set of all upper distribution functions. We shall denote the upper distribution function $\mathcal{U}(p, q)$ by $M_{p, q}(x)$, whence $M_{p, q}(x)$ will denote the value of $M_{p, q}$ at $x \in \mathbb{R}$.

An example of upper chaos transversal space is an upper probabilistic space which is a nonempty set $X$ together with the functions $M_{p, q}(x)$ with the following properties: $M_{p, q}(x)=M_{q, p}(x), M_{p, q}(0)=1$,

$$
M_{p, q}(x)=0 \text { for } x>0 \text { if and only if } p=q \text {; }
$$

and if there is nondecreasing function $\mu:[0,1]^{2} \rightarrow[0,1]$ with the property $\mu(t, t) \leq t$ for all $t \in[0,1]$ such that

$$
M_{p, q}(x+y) \leqslant \mu\left(M_{p, r}(x), M_{r, q}(y)\right)
$$

for all $p, q, r \in X$ and for all $x, y \geqslant 0$. Then, from (4), we immediately obtain that every upper probabilistic space, for $\rho[p, q]=M_{p, q}(x): X \times X \rightarrow[0,1]$ is an upper chaos transversal space.

Example 7. (Upper parametric transversal chaos spaces). In connection with the preceding facts, the function $M: X \times X \times \mathbb{R} \rightarrow[a, b]$ for some $-\infty \leq a<b \leq$ $+\infty$ is called an upper parametric chaos transverse on $X$ (or upper parametric chaos transversal) iff: there is $c \in \mathbb{R}_{+}^{0}$ such that $M(u, v, t)=a$ for every $t>c$ if and only if $u=v$, and $\lim _{n \rightarrow \infty} M\left(u, v, x_{n}\right)=a$ for arbitrary nondecreasing sequence $\left\{x_{n}\right\}_{n \in \mathbb{N}}$ in $[c,+\infty)$ with $x_{n} \rightarrow+\infty(n \rightarrow \infty)$.

A transversal upper parametric chaos space is a set $X$ together with a given upper parametric chaos transverse $M: X \times X \times \mathbb{R} \rightarrow[a, b]$ for some $-\infty \leq a<b \leq+\infty$ in notation $X:=(X, M)$. De facto, every transversal upper parametric chaos space $X:=(X, M)$, for $A=M$, is an upper chaos transversal space. For this spaces the following "asymptotic" statement holds as a consequence of Theorem 3.

Corollary 7. Let $X:=(X, M)$ be a transversal upper parametric chaos space with the continuous upper parametric chaos transverse $M \in \mathbb{R}_{+}^{0}$, $T: X \rightarrow X$ is a continuous function, and $X$ with the condition of upper chaos TCS-convergence. Suppose that there exists a function $\varphi:[c,+\infty) \rightarrow$ $[c,+\infty)$ for some $c \in \mathbb{R}$ satisfying $(A s)$ and $\varphi(t)>t$ for every $t>c$, and such that

$$
M\left(T^{n}(x), T^{n}(y), t\right) \leq M\left(x, y, \varphi^{n}(t)\right) \text { for every } n \in \mathbb{N},
$$

for every $t>c$, and for all $x, y \in X$. Then $T$ has a unique fixed point in $X$. 
Proof. (Application of Theorem 3). We define a function $A: X \times X \rightarrow[a, b]$ for some $-\infty \leq a<b \leq+\infty$ by $A(u, v):=M(u, v, t)$ and define a sequence of functions $\left\{\alpha_{n}(u, v)\right\}_{n \in \mathbb{N}}$ by $\alpha_{n}(u, v):=M\left(u, v, \varphi^{n}(t)\right)$. Thus we obtain the following fact that $\alpha_{n}(u, v) \rightarrow \lim _{n \rightarrow \infty} M\left(u, v, \varphi^{n}(t)\right)=a$ as $n \rightarrow \infty$ as in Theorem 3. On the other hand $x \mapsto A(x, T x):=M(x, T x, t)$ is $T$ orbitally lower semicontinuous, because $T$ and $M$ are continuous. Since $A(u, v):=M(u, v, t)=a$ implies $u=v$ for every $t>c$, and since $X$ satisfies the condition of upper chaos TCS-convergence, applying Theorem 3 we obtain that $T$ has a unique fixed point in $X$. The proof is complete.

As a special case of Corollary 7 we obtain the following "asymptotic" statement for transversal upper parametric chaos spaces.

Corollary 8. Let $X:=(X, M)$ be a transversal upper parametric chaos space with the continuous upper parametric chaos transverse $M \in \mathbb{R}_{+}^{0}$, $T: X \rightarrow X$ is a continuous function, and $X$ with the condition of upper chaos TCS-convergence. Suppose that there exists an increasing continuous function $\varphi:[c,+\infty) \rightarrow[c,+\infty)$ for some $c \in \mathbb{R}$ satisfying $\varphi(t)<t$ for every $t \in[c,+\infty)$ such that

$$
M\left(T^{n}(x), T^{n}(y), \varphi^{n}(t)\right) \leq M(x, y, t) \text { for every } n \in \mathbb{N},
$$

for every $t>c$, and for all $x, y \in X$. Then $T$ has a unique fixed point in $X$.

Proof. Since for the function $\varphi:[c,+\infty) \rightarrow[c,+\infty)$ for some $c \in \mathbb{R}$ there is its inverse function $\varphi^{-1}:[c,+\infty) \rightarrow[c,+\infty)$ with the property (As), thus by $(\mathrm{F})$ we obtain

$$
M\left(T^{n}(x), T^{n}(y), t\right) \leqslant M\left(x, y, \varphi^{-n}(t)\right) \text { for } n \in \mathbb{N},
$$

and for all $x, y \in X$, i.e., this means that a form of inequality (R) holds. Thus, applying Corollary 7 we obtain this statement as a consequence. The proof is complete.

An essential remark. We notice that the upper parametric transversal chaos spaces are, de facto, also the upper transversal chaos spaces.

\section{REFERENCES}

[1] A. Appert and Ky Fan, Espaces topologiques intermédiaires. Problème de la distanciation, Actuelités scient., Paris, 1951.

[2] P. Azzimondi and C. Scaravelli, Un teorema del punto unito in spazi metrici generalizzati, Riv. Mat. Univ. Parma, 5 (1979), 773-780.

[3] G. Bocsan and G. Constantin, The Kuratowski function and some applications to probabilistic metric spaces, Atti dela Academia Nazionale dei Lincei, 8 (1973), 236240.

[4] S. Czerwik, Nonlinear set-valued contraction mapping in b-metric spaces, Atti Sem.Mat.Fis.Univ.Modena, 46 (1998), 263-276. 
[5] E. W. Chittenden, On the equivalence of ecart and voisinage, Trans. Amer. Math. Soc., 18 (1917), 161-166.

[6] M. Cicchese, Distanze generalizzate uniformemente continue, Riv. Mat. Univ. Parma, 3 (1974), 133-137.

[7] L. Collatz, Funktionalanalysis und Numerische Mathematik, Springer-Verlag, Berlin, 1964.

[8] J. Colmez, Espaces à écart généralisé régulier, C. R. Acad. Sci. Paris, 224 (1947), p.p. 372 .

[9] G. Constantin and I. Istrăţescu, Elements of Probabilistic Analysis, Kluwer Academic Publishers, 1989.

[10] R. Doss, Sur la condition de régularité pour l'ecart abstrait. Écart abstrait symétrique et régulier, C. R. Acad. Sci. Paris, 223 (1946), 14-16, 1087-1088.

[11] M. Fréchet, La notion d'écart et le calcul fonctionnel, C. R. Acad. Sci. Paris, 140 (1905), 772-774.

[12] M. Fréchet, Sur quelques points du calcul fonctionnel, Thèse, Paris, 1905. Rendiconti Circolo Mat. Palermo, 22 (1906), 1-74.

[13] A. H. Frink, Distance functions and the metrization problem, Bull. Amer. Math. Soc., 43 (1937), 133-142.

[14] V. Istrăţescu, Introducere in teoria spatiilor metrice probabilistic applicatti, Edifura Technica, Bucarest, 1974.

[15] A. A. Ivanov, Isledovanii po topologii-II, Zapiski naučnih seminarov lomi, Lenjingrad 66 (1976), 5-102.

[16] O. Kaleva and S. Seikkala, On fuzzy metric spaces, Fuzzy Sets and Systems, 12 (1984), 215-229.

[17] W. A. Kirk, Fixed points of asymptotic contractions, J. Math.Anal.Appl., 277 (2003), 645-650.

[18] Đ. R. Kurepa, Tableaux ramifiés d'ensembles. Espaces pseudo-distanciés, C. R. Acad. Paris, 198 (1934), 1563-1565.

[19] Đ. R. Kurepa, Ensembles ordonnés et ramifiés, Thèse, Paris, 1935, p.p. 1-138; Publ. Inst. Math. Belgrade, 4 (1935), 1-138.

[20] Đ. R. Kurepa, Sur les classes $(E)$ et $(D)$, Publ. Inst. Math. Belgrade, 55 (1936), 124-132.

[21] Đ. R. Kurepa, Un critère de distanciabilitè, Mathematica (Cluj), 13 (1937), 59-65.

[22] Đ. R. Kurepa, Distanca numerica. Distanca non numerica, Publ. Seminario Matematica, Bari, 1963.

[23] Đ. R. Kurepa, On the existence of pseudometric non totally orderable spaces, Glasnik Mat. Fiz. Astr. Zagreb, 18 (1963), 183-194.

[24] K. Menger, Untersuchungen über allgemeine Metric, Math. Annalen, 100 (1928), $75-163$.

[25] K. Menger, Statistical metrics, Proc. Nat. Acad. Sci., USA, 28 (1942), 535-537.

[26] O. Onicescu, Nombres et Systèmes Aléatoires, Éditions de L' Académie de la R. P. Roumaine, Bucarest, 1964.

[27] P. Papić, Sur une classe d'espaces abstrait, Glasnik Mat. Fiz. Astr. Zagreb, 9 (1954), 197-216.

[28] B. Schweizer and A. Sklar, Statistical metric spaces, Pacific J. Math., 10 (1960), $313-334$. 
[29] V. M. Sehgal, Some fixed point theorems in functional analysis and probability, Ph. D. dissertation, Wayne State Univ., 1966.

[30] V. M. Sehgal and A. T. Bharucha-Reid, Fixed points of Contraction Mappings on Probabilistic Metric Spaces, Math. Systems Theory, 6 (1972), 97-102.

[31] H. Sherwood, Somplete Probabilistic Metric Spaces, Z. Wahrscheinlichkeitstheorie verw. Geb., 20 (1971), 117-128.

[32] R. Sikorski, Remark on some topological spaces of high power, Fund. Math., 37 (1950), 125-136.

[33] A. N. Šerstnev, On the probabilistic generalization of metric spaces, Kazan. gosudarst. Univ., učenye Zapiski, 124 (1967), 109-119.

[34] M. R. Tasković, Some new principles in fixed point theory, Math. Japonica, 35 (1990), 645-666.

[35] M. R. Tasković, Nonlinear Functional Analysis, Fundamental elements of theory, First Book. Monographs, Zavod za udžbenike i nastavna sredstva, Beograd 1993, 792 p.p. (Serbo-Croation). English summary, Comments only new main results of this book. Vol. 1 (1993), 713-752.

[36] M. R. Tasković, Transversal spaces, Math. Moravica, 2 (1998), 133-142.

[37] M. R. Tasković, Transversal intervally spaces, Math. Moravica, 7 (2003), 91-106.

[38] M. R. Tasković, Furcate points and lower BCS-convergence in the fixed point theory, First International Videoconference of Math. Science - Fixed Point Theory, Japanese Assoc. Math. Sciences, Osaka, Japan, 2003, 10 p.p.

[39] M. R. Tasković, Nonlinear Functional Analysis, Second Book, Monographs - Global Convex Analysis: General convexity, Variational methods and Optimization, Zavod za udžbenike i nastavna sredstva and Vojnoizdavački zavod, Beograd 2001, (In Serbian), 1223 pages.

[40] M. R. Tasković, Theory of transversal point, spaces, and forks - Fundamental Elements and Applications, Monographs of a new mathematical theory, VIZ - Beograd 2005, (In Serbian), 1054 pages, English summary: 1001-1022.

[41] M. R. Tasković, Fixed points on transversal edges spaces, Math Moravica 7 (2003), 175-186.

[42] M. R. Tasković, Survey on transversal normed spaces, Math Moravica 7 (2003), 153174.

[43] S. Todorčević, On a theorem of $Đ$. Kurepa, in Topology and order structures I, Math. Centre Tracts 142, Amsterdam, 1981, 173-176.

[44] A. Wald, On a statistical generalization of metric spaces, Proc. Nat. Acad. Sci., U.S.A., 29 (1943), 196-197.

Milan R. TAsković

Faculty of Mathematics

11000 Belgrade, P.O. Box 550

SERBIA

Home Address:

Milan R. Tasković

Nehruova 236

11070 Belgrade

Serbia

E-mail address: andreja@predrag.us 